\title{
PREVALÊNCIA DA HIPERTENSÃO ARTERIAL E DIABETES MELLITUS NAS ESTRATÉGIAS DE SAÚDE DA FAMÍLIADE UM MUNICÍPIO NO INTERIOR PAULISTA
}

\author{
Nathália Serafim da Silva, Viviane de Freitas Cardoso, Ana Lúcia de Jesus Almeida \\ ${ }^{2}$ Universidade Estadual Paulista - UNESP, Departamento de Fisioterapia, Presidente Prudente, São Paulo. E-mail: \\ Nathaliaserafim.s@hotmail.com
}

\section{RESUMO}

A Hipertensão Arterial e Diabetes Mellitus são doenças crônico-degenerativas que mais atingem a população e o Programa HiperDia é uma estratégia de controle e acompanhamento. O objetivo desse trabalho foi realizar um estudo epidemiológico entre asESFs, com foco nessas doenças. A metodologia constou da análise descritiva dos dados consolidados denovembro/2013 e dezembro/2015, fornecidos pela SMS do município de Presidente Prudente. Para analise estatística foi utilizado o programa informático Statistical Program for Social Sciences (SPSS) para: teste Kolmogorov-Smirnov e coeficiente de correlação de Pearson. Em todos os testes estatísticos utilizados foi considerado um nível de significância de $5 \%$. Os resultados mostraram que o aumento da incidência das doenças foi de $+0,42 \%$ para DIA e $+0,54 \%$ para HA. Concluímos que o aumento do envelhecimento da população está associado com o aumentos dessas doenças e que estudos como este permite uma abordagem mais contextualizada.

Palavras-chave: Estratégia de Saúde da Família, Atenção Básica, Análise epidemiológica, Hipertensão, Diabetes.

\section{PREVALENCE OF HYPERTENSION AND DIABETES MELLITUS BLOOD ON STRATEGIES FAMÍLIADE HEALTH A MUNICIPALITY IN THE BRASILIAN STATE OF SÃO PAULO}

\begin{abstract}
Arterial Hypertension and Diabetes Mellitus are chronic degenerative diseases that affect the population and the HiperDia program is a control and monitoring strategy. The aim of this study was an epidemiological study among the ESFs, with focus on those diseases. The methodology consisted of descriptive analysis of the consolidated data for November / 2013 and December / 2015, supplied by SMS in Presidente Prudente. For statistical analysis we used the Statistical Program for Social Sciences (SPSS) for: Kolmogorov -Smirnov test and Pearson correlation. In all statistical tests it was considered a significance level of $5 \%$. The results showed that the increased incidence of the disease was $+0.42 \%$ to $+0.54 \%$ DIA and HA. We conclude that the increase of population aging is associated with the increase of these diseases and that studies like this allows a more contextualized approach. Keywords: Family Health Strategy, Primary Care, epidemiological analysis, hypertension, diabetes.
\end{abstract}




\section{INTRODUÇÃO}

A Hipertensão Arterial e Diabetes Mellitus são doenças crônico-degenerativas, e apresentam aspectos em comum, como: etiopatogenia, fatores de risco, ocorrência geralmente assintomática, importância do tratamento não medicamentoso, complicações e necessidade de controle permanente (RIBEIRO, et al 2014). Diante do aumento dessas intercorrências foi criado pelo Ministério da Saúde, em 2002, o Programa Nacional de Hipertensão e Diabetes Mellitus - HiperDia (CARVALHO, 2012).

A Estratégia de Saúde da Família (ESF) é modelo assistencial que, a partir da atenção básica, utiliza de diversas políticas de cuidado, dentre elas o HiperDia, Rede Cegonha, Melhor em Casa, Programa de Controle do Tabagismo, dentre outras (SANTOS et al, 2011, BRASIL, 2016) a fim de acompanhar seus usuários, buscando maior participação, adesão, resolutividade e impacto na situação de saúde e coletividade.

A análise epidemiológica das condições de saúde da população atendida nas ESFs é importante para o estabelecimento de prioridades, a alocação de recursos e a orientação programática, a fim de implantar intervenções que sejam mais eficientes e o presente estudo tem esse enfoque.

\section{OBJETIVOS:}

Realizar um estudo epidemiológico e observar as correlações entre as condições de saúde das ESFs do município de Presidente Prudente, com foco na Hipertensão Arterial e Diabetes Mellitus, nos anos de 2013 e 2015.

\section{METODOLOGIA:}

Os dados analisados neste estudo são oriundos do Sistema de Informação de Atenção Básica (SIAB) e fornecidos pela Secretaria Municipal de Saúde - SMS de Presidente Prudente, no formato dos consolidados do SIAB do mês de novembro de 2013, quando existiam 15 unidades de ESF no município e do mês de dezembro de 2015, somando 18 unidades de ESF, pois no período houve o acréscimo de três unidades de ESF no município.

As variáveis selecionadas no consolidado foram: o número de pessoas e famílias cadastradas pelas ESF; a faixa etária e a prevalência de Hipertensão Arterial e Diabetes Mellitus. Os dados foram tabulados e realizada a análise descritiva da média,do desvio padrão, dos percentuais e o das diferenças entre os anos estudados $(\Delta)$. Para análise estatística foi utilizado o programa informático Statistical Program for Social Sciences (SPSS), nele foi realizado o teste Kolmogorov-Smirnov para avaliar normalidade e depois calculado do coeficiente de correlação de Pearson e de Spearman's de acordo com a normalidade das variáveis. Em todos os testes estatísticos utilizados foi considerado um nível de significância de $5 \%(p<0,05)$. Este estudo foi avaliado pelo Comitê de Ética em Pesquisa da Faculdade de Ciências e Tecnologia - UNESP - Presidente Prudente e aprovado em 23 de maio de 2015 (CAAE: 41034914.1.0000.5402).

\section{RESULTADOS}

Atualmente o município de Presidente Prudente tem 18 estratégias de saúde da família (ESF). A tabela 1 mostra que na maioriadas ESFs houve redução de pessoas e famílias no período analisado, sendo apenas a ESF Primavera que aumentou em ambas as variáveis. 
Tabela 1. Distribuição da população das ESFs do Município de Presidente Prudente

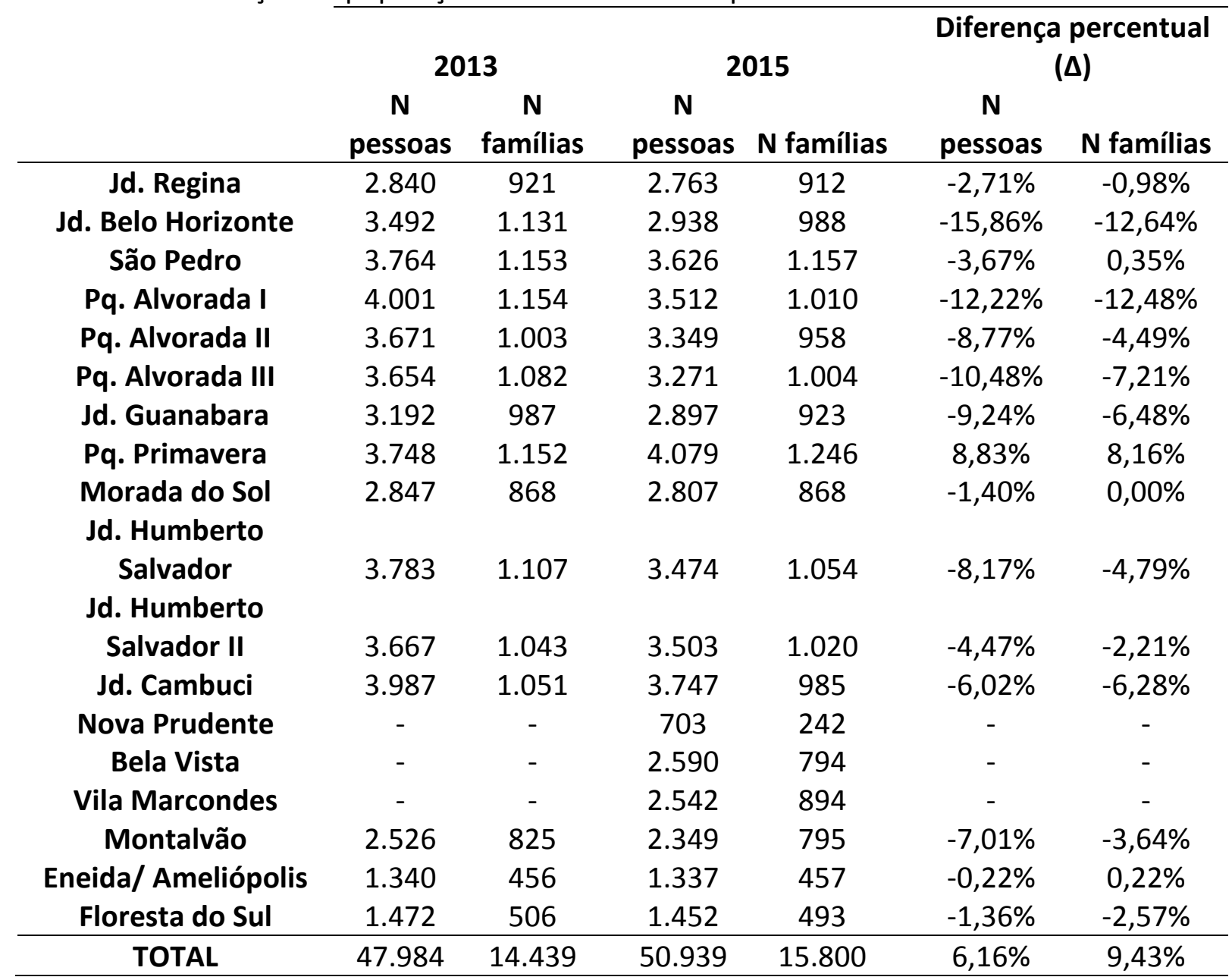

Fonte: SIAB 2013/2015

No SIAB a distribuição por faixas etárias é da seguinte maneira: <1 ano; 1 a 9; 10 a 19; 20 a 39; 40 a 49; 50 a 59 anos e $>60$ anos. Na tabela 2 as faixas etárias foram agrupadas, proporcionalmente em quartis de 20 anos, a fim de caracterizar a população entre crianças e adolescentes; adultos jovens; adultos de 40 a 59 anos e idosos. 
Tabela 2. Percentual por faixa etária entre os anos 2013 e 2015

\begin{tabular}{|c|c|c|c|c|c|c|c|c|}
\hline & \multicolumn{2}{|c|}{$<1$ a 19} & \multicolumn{2}{|c|}{20 a 39} & \multicolumn{2}{|c|}{40 a 59} & \multicolumn{2}{|c|}{$60>$} \\
\hline & 2013 & 2015 & 2013 & 2015 & 2013 & 2015 & 2013 & 2015 \\
\hline & $\%$ & $\%$ & $\%$ & $\%$ & $\%$ & $\%$ & $\%$ & $\%$ \\
\hline Jd.Regina & 27,56 & 26,15 & 32,32 & 31,16 & 25,28 & 25,58 & 14,82 & 17,08 \\
\hline Jd. Belo Horizonte & 25,96 & 23,27 & 30,15 & 28,08 & 25,68 & 26,37 & 18,18 & 22,49 \\
\hline São Pedro & 28,42 & 27,39 & 29,8 & 29,43 & 26,01 & 24,95 & 15,75 & 18,2 \\
\hline Pq. Alvorada I & 28,6 & 27,07 & 31,51 & 30,52 & 27,52 & 28,35 & 12,34 & 14,03 \\
\hline Pq. Alvorada II & 33,7 & 30,57 & 33,15 & 33,14 & 23,59 & 25,19 & 9,53 & 11,07 \\
\hline Pq. Alvorada III & 26,9 & 24,96 & 30,95 & 29,17 & 25,12 & 26,86 & 16,0 & 18,98 \\
\hline Jd. Guanabara & 30,91 & 28,8 & 32,42 & 31,72 & 23,5 & 24,91 & 13,15 & 14,53 \\
\hline Pq. Primavera & 27,87 & 26,71 & 32,36 & 33,88 & 26,97 & 25,19 & 12,78 & 14,19 \\
\hline Morada do Sol & 37,18 & 35,54 & 30,63 & 30,57 & 22,34 & 23,22 & 9,83 & 10,83 \\
\hline Jd. Humberto Salvador & 35,32 & 35,21 & 33,54 & 32,73 & 25,03 & 27,33 & 6,0 & 7,74 \\
\hline Humberto Salvador II & 35,79 & 32,93 & 32,1 & 31,2 & 24,29 & 27,05 & 7,7 & 8,79 \\
\hline Jd. Cambuci & 35,83 & 31,99 & 33,48 & 34,48 & 22,95 & 24,04 & 7,7 & 9,47 \\
\hline Nova Prudente & & 29,57 & & 29,73 & & 24,46 & & 16,21 \\
\hline Bela Vista & & 39,86 & & 34,86 & & 18,56 & & 6,67 \\
\hline Vila Marcondes & & 23,16 & & 27,18 & & 24,81 & & 24,82 \\
\hline Montalvão & 27,43 & 25,7 & 29,1 & 28,44 & 26,08 & 27,75 & 17,22 & 18,09 \\
\hline Eneida/Ameliópolis & 28,64 & 26,83 & 28,36 & 26,93 & 26,19 & 27,81 & 16,79 & 18,39 \\
\hline Floresta do Sul & 26,14 & 24,29 & 29,55 & 28,44 & 26,08 & 27,12 & 18,2 & 20,11 \\
\hline Total & 31,14 & 29,2 & 31,9 & 31,36 & 25,31 & 25,8 & 12,65 & 14,67 \\
\hline
\end{tabular}

Fonte: SIAB 2013/2015

A média da incidência nas ESFs do município foi para a Hipertensão Arterial (HAS) $15,56 \% \pm$ 4,15, em 2013, evoluindo para 16,02\% $\pm 4,15$, em 2015, e Diabetes Mellitus (DIA) foi de 4,36\% $\pm 1,08$ evoluindo para $4,73 \% \pm 1,30$.

Na tabela 3 podemos observar a incidência total das doenças por bairro, além da diferença percentual no período estudado. Na Diabetes Mellitus, em 2013, oito unidades (53,3\%) apresentavam índices acima da média do município para essa comorbidade e, em 2015, nove unidades (50\%). Enquanto na Hipertensão Arterial apresentava, em 2013, oito unidades (53,3\%) acima da média de incidência de Hipertensão Arterial no município para essa população e, em 2015, nove unidades $(55,56 \%)$. 
Tabela 3. Prevalência das intercorrências referidas no ano de 2013 e 2015

\begin{tabular}{|c|c|c|c|c|c|c|c|c|c|c|}
\hline \multirow{3}{*}{ Jd.Regina } & \multicolumn{5}{|c|}{ Diabetes Mellitus } & \multicolumn{5}{|c|}{ Hipertensão Arterial } \\
\hline & \multicolumn{2}{|c|}{2013} & \multicolumn{2}{|c|}{2015} & \multirow{2}{*}{$\begin{array}{c}\Delta \\
0,30 \%\end{array}$} & \multicolumn{2}{|c|}{2013} & \multicolumn{2}{|c|}{2015} & \multirow{2}{*}{$\begin{array}{c}\Delta \\
0,39 \%\end{array}$} \\
\hline & 121 & $4,26 \%$ & 126 & $4,56 \%$ & & 478 & $16,80 \%$ & 475 & $17,19 \%$ & \\
\hline Jd. Belo Horizonte & 230 & $6,59 \%$ & 219 & $7,45 \%$ & $0,86 \%$ & 716 & $20,50 \%$ & 640 & $21,78 \%$ & $1,28 \%$ \\
\hline São Pedro & 178 & $4,73 \%$ & 225 & $6,21 \%$ & $1,48 \%$ & 586 & $15,60 \%$ & 632 & $17,43 \%$ & $1,83 \%$ \\
\hline Pq. Alvorada I & 181 & $4,52 \%$ & 180 & $5,13 \%$ & $0,61 \%$ & 626 & $15,70 \%$ & 581 & $16,54 \%$ & $0,84 \%$ \\
\hline Pq. Alvorada II & 140 & $3,81 \%$ & 129 & $3,85 \%$ & $0,04 \%$ & 496 & $13,50 \%$ & 445 & $13,29 \%$ & $-0,21 \%$ \\
\hline Pq. Alvorada III & 174 & $4,76 \%$ & 171 & $5,23 \%$ & $0,47 \%$ & 600 & $16,40 \%$ & 562 & $17,18 \%$ & $0,78 \%$ \\
\hline Jd. Guanabara & 156 & $4,89 \%$ & 150 & $5,18 \%$ & $0,29 \%$ & 438 & $13,70 \%$ & 401 & $13,84 \%$ & $0,14 \%$ \\
\hline Pq. Primavera & 107 & $2,85 \%$ & 109 & $2,67 \%$ & $-0,1$ & 474 & $12,70 \%$ & 495 & $12,14 \%$ & $-0,56 \%$ \\
\hline Morada do Sol & 114 & $4,00 \%$ & 117 & $4,17 \%$ & $0,17 \%$ & 358 & $12,60 \%$ & 331 & $11,79 \%$ & $-0,81 \%$ \\
\hline Jd. Humberto Salvador & 106 & $2,80 \%$ & 135 & $3,89 \%$ & $1,09 \%$ & 370 & $9,78 \%$ & 415 & $11,95 \%$ & $2,17 \%$ \\
\hline Humberto Salvador II & 132 & $3,60 \%$ & 123 & $3,51 \%$ & $-0,09 \%$ & 414 & $11,30 \%$ & 431 & $12,30 \%$ & $1,00 \%$ \\
\hline Jd. Cambuci & 114 & $2,86 \%$ & 125 & $3,34 \%$ & $0,48 \%$ & 443 & $11,10 \%$ & 415 & $11,08 \%$ & $-0,02 \%$ \\
\hline Nova Prudente & - & - & 25 & $3,56 \%$ & - & - & - & 118 & $16,79 \%$ & - \\
\hline Bela Vista & - & - & 83 & $3,20 \%$ & - & - & - & 270 & $10,42 \%$ & - \\
\hline Vila Marcondes & - & - & 161 & $6,33 \%$ & - & - & - & 539 & $21,20 \%$ & - \\
\hline Montalvão & 125 & $4,95 \%$ & 134 & $5,70 \%$ & $0,75 \%$ & 445 & $17,60 \%$ & 422 & $17,97 \%$ & $0,37 \%$ \\
\hline Eneida/Ameliópolis & 76 & $5,67 \%$ & 78 & $5,83 \%$ & $0,16 \%$ & 323 & $24,10 \%$ & 316 & $23,64 \%$ & $-0,46 \%$ \\
\hline Floresta do Sul & 76 & $5,16 \%$ & 78 & $5,37 \%$ & $0,21 \%$ & 324 & $22,00 \%$ & 317 & $21,83 \%$ & $-0,17 \%$ \\
\hline Total & 2030 & $4,23 \%$ & 2368 & $4,65 \%$ & $0,42 \%$ & 7091 & $14,78 \%$ & 7805 & $15,32 \%$ & $0,54 \%$ \\
\hline
\end{tabular}

Fonte: SIAB 2013/2015

$\mathrm{Na}$ análise estatística e correlação observamos que o número de pessoas acima de 60 anos correlaciona-se com o número de Diabéticos, tanto em 2013 ( $r=0,84, p=0,001)$, como em 2015 ( $r=$ $0,79, p=0,001)$, ambas apresentando uma correlação forte. Porém a diferença entre os anos, nessas variáveis, não apresentou significância ( $r=0,39, p=0,151)$.

Para a Hipertensão Arterial o aumento de pessoas acima de 60 anos no período entre o ano de $2013(r=0,91, p=0,00)$ e $2015(r=0,90, p=0,000)$ apresentou uma correlação muito forte. $E$ a diferença entre os anos nessas variáveis apresentou significância e uma correlação moderada ( $r$ $=0,539, p=0,038$ ). Esses dados mostram que a população idosa influencia de maneira diretamente proporcional na prevalência da Hipertensão Arterial.

\section{DISCUSSÃO}

A tabela 2 nos mostra que do ano de 2013 a 2015 aumentou o número de idosos maiores de 60 anos em todas as ESFs analisadas e o número de crianças menores de um ano e adolescentes até 19 anos diminuiu em todas as ESFs, fato que pode ser explicado pela transição demográfica atual, onde aumentou o número de idosos e diminuiu a taxa de natalidade (BRASIL, 2006), bem como a mobilidade dentro do município.

As ESFs que apresentaram maior proporção de indivíduos acima de 60 anos (Vila Marcondes, Belo Horizonte, Floresta do Sul) foram também as ESFs que apresentaram maior número de hipertensos, evidenciando a relação entre essa patologia e a idade. No estudo de Cipullo, a prevalência da hipertensão na população assistida foi de $25,3 \%$ com um aumento progressivo com a idade, chegando a $70 \%$ entre os indivíduos com mais de 70 anos. Além disso, fatores socioeconômicos também tem um importante papel nas condições de saúde e influenciam no acesso ao sistema de saúde, no entendimento da condição clinica, grau de informação e aderência ao tratamento (CIPULLO et al, 2010). 
O acelerado ritmo do processo de envelhecimento da população, combinados com a grande tendência à hábitos inadequados de alimentação e o sedentarismo, além de mudanças sóciocomportamentais, colaboram com o crescimento da incidência e prevalência da Diabetes Mellitus, assim como a mortalidade (FRANCISCO et al, 2010).De acordo com os dados da Pesquisa Nacional de Saúde, realizada pelo Ministério da Saúde em parceria com o IBGE, há uma prevalência da patologia de 6,2\% para a população adulta (BRASIL, 2015), enquanto que no município de Presidente Prudente essa prevalência é de 4,65\%, (tabela 3). Entretanto, a prevalência oscila de 2,67 até 7,45\%.

Essas doenças apresentam uma forte correlação com o aumento de pessoas acima de 60 anos segundo o teste de Pearson, informação que merece uma análise mais aprofundada, tendo em vista que em algumas unidades foi possível observar decréscimo na incidência da patologia mesmo com o aumento da população acima de 60 anos. Na correlação de Pearson as diferenças entre os anos, quando analisado o $\Delta>60$ anos e as doenças tem-se uma correlação moderada para o $\Delta$ Hipertensão Arterial e sem significância para o $\Delta$ Diabetes Mellitus. 0 que pode apontar para diferenças na abordagem e ou adesão da população às orientações e hábitos de saúde.

O estudo tem limitações por trabalhar com os dados já consolidados não permitindo aprofundar a análise dos dados sobre o comportamento epidemiológico dentro de cada faixa etária. Para tal se faz necessário uma investigação em cada unidade de ESF. Uma abordagem mais qualitativa para entender os motivos que provocaram diferenças na incidência da Hipertensão Arterial e Diabetes Mellitus entre os anos estudados deve qualificar e aprofundar este estudo, além de contribuir para o entendimento e maior controle dessas variáveis.

\section{CONCLUSÃO}

O estudo permite apresentar as diferenças demográficas e epidemiológicasem relação a Hipertensão Arterial e Diabetes Mellitus nas 18 ESFs do município de Presidente Prudente. Na maioria dos bairros fica evidente o aumento do envelhecimento da população associado com o aumentos dessas doenças. Estudos como este permitem um olhar crítico para os dados com possibilidade de uma abordagem mais contextualizada.

\section{REFERÊNCIAS}

BRASIL. Ministério da Saúde.Atenção Básica. Secretaria de Atenção à Saúde, Política Nacional de Humanização. - Brasília : Ministério da Saúde, 2010.

BRASIL. Ministério da Saúde. Diabetes atinge 9 milhões de brasileiros, 2015. Disponível em: http://www.brasil.gov.br/saude/2015/07/diabetes-atinge-9-milhoes-de-brasileiros.

BRASIL. Ministério da Saúde. Ações e Programas, 2016. Disponível em: http://portalsaude.saude.gov.br/index.php/cidadao/acoes-e-programas

BRASIL. Ministério da Saúde. Secretaria de Atenção à Saúde. Departamento de Atenção Básica. Envelhecimento e saúde da pessoa idosa. Brasília: Ministério da Saúde, 2006. 192 p. (Normas e Manuais Técnicos. Cadernos de Atenção Básica, n.19, Série A).

CIPULLO, J.P., MARTIN, J.F.V, CIORLIA, L.A.D.S. et al. Prevalência e fatores de risco para hipertensão em uma população urbana brasileira.ArqBrasCardiol, 94(4), 519-26, 2010. https://doi.org/10.1590/S0066-782X2010005000014

DIAS, Ana Cristina Garcia; TEIXEIRA, Marco Antônio Pereira. Gravidez na adolescência: um olhar sobre um fenômeno complexo. Paidéia (Ribeirão Preto), Ribeirão Preto , v. 20, n. 45, p. 123131, Apr. 2010. 
FRANCISCO, P.M.S.B et al. Diabetes auto-referido em idosos: Prevalência, fatores associados e práticas de controle. Cad. Saúde Pública, v. 26, n.1, p. 175-184, 2010.

RIBEIRO, M., FARIA, L., LEMOS, G.. Atenção Farmacêutica em Pacientes com Hipertensão Arterial Sistêmica em uma Unidade de Saúde de Jequié-BA. JMPHC. Journal of Management and Primary Health Care, América do Norte, 4, fev. 2014. Disponível em: (http://imphc.com/ojs/index.php/01/article/view/176/109). Acesso em: 26 Fev. 2014.

SANTOS, Karina Tonini et al. Agente comunitário de saúde: perfil adequado a realidade do Programa Saúde da Família. Ciência \& Saúde Coletiva, v. 16, n. 1, p. 1023-1028, 2011. 